\title{
New inhalation-optimized itraconazole nanoparticle-based dry powders for the treatment of invasive pulmonary aspergillosis
}

This article was published in the following Dove Press journal:

International Journal of Nanomedicine

17 October 2012

Number of times this article has been viewed

\section{Christophe Duret ${ }^{\prime}$ \\ Nathalie Wauthoz' \\ Thami Sebti ${ }^{2}$ \\ Francis Vanderbist ${ }^{2}$ \\ Karim Amighi'}

'Laboratoire de Pharmacie Galénique et de Biopharmacie, Université Libre de Bruxelles, Brussels, Belgium; ${ }^{2} \mathrm{SMB}$ Laboratoires, Brussels, Belgium
Correspondence: Christophe Duret Université Libre de Bruxelles (ULB), Boulevard du triomphe campus de la plaine (accès 2), Service de pharmacie galénique et biopharmacie,

bat $\mathrm{BC}$ niveau 6, Brussels, Belgium

$\mathrm{Tel}+326505331$

Fax +326505269

Email cduret@ulb.ac.be
Purpose: Itraconazole (ITZ) dry powders for inhalation (DPI) composed of nanoparticles (NP) embedded in carrier microparticles were prepared and characterized.

Methods: DPIs were initially produced by reducing the ITZ particle size to the nanometer range using high-pressure homogenization with tocopherol polyethylene 1000 succinate (TPGS, $10 \% \mathrm{w} / \mathrm{w}$ ITZ) as a stabilizer. The optimized nanosuspension and the initial microsuspension were then spray-dried with different proportions of or in the absence of mannitol and/or sodium taurocholate. DPI characterization was performed using scanning electron microscopy for morphology, laser diffraction to evaluate the size-reduction process, and the size of the dried NP when reconstituted in aqueous media, impaction studies using a multistage liquid impactor to determine the aerodynamic performance and fine-particle fraction that is theoretically able to reach the lung, and dissolution studies to determine the solubility of ITZ.

Results: Scanning electron microscopy micrographs showed that the DPI particles were composed of mannitol microparticles with embedded nano- or micro-ITZ crystals. The formulations prepared from the nanosuspension exhibited good flow properties and better fine-particle fractions, ranging from $46.2 \% \pm 0.5 \%$ to $63.2 \% \pm 1.7 \%$ compared to the $23.1 \% \pm 0.3 \%$ that was observed with the formulation produced from the initial microsuspension. Spray-drying affected the NP size by inducing irreversible aggregation, which was able to be minimized by the addition of mannitol and sodium taurocholate before the drying procedure. The ITZ NP-based DPI considerably increased the ITZ solubility ( $58 \pm 2$ increased to $96 \pm 1 \mathrm{ng} / \mathrm{mL}$ ) compared with that of raw ITZ or an ITZ microparticle-based DPI $(<10 \mathrm{ng} / \mathrm{mL})$.

Conclusion: Embedding ITZ NP in inhalable microparticles is a very effective method to produce DPI formulations with optimal aerodynamic properties and enhanced ITZ solubility. These formulations could be applied to other poorly water-soluble drugs and could be a very effective alternative for treating invasive pulmonary aspergillosis.

Keywords: aspergillosis, spray-drying, homogenization, inhalation, saturation, solubility

\section{Introduction}

Invasive pulmonary aspergillosis is an opportunistic disease that primarily occurs in immunocompromised patients. ${ }^{1}$ Despite current intravenous/oral prophylactic and therapeutic treatments, the incidence of this pathology is very high in the immunocompromised population and possesses a mortality rate of $40 \%-90 \%$ when the invasive state is reached. ${ }^{2}$ The localized delivery of an antifungal drug to the lung by nebulization in different murine models of pulmonary invasive aspergillosis has already been proved to enhance therapeutic outcomes compared to oral therapy. ${ }^{3}$ Indeed, this mode of administration should allow for an increased concentration of the antifungal drug at the 
site of infection while reducing systemic concentrations. Such a reduction would minimize systemic side effects and the drug-drug interactions that are often problematic with antifungal drugs. Given this potential, the development of dry powders for inhalation (DPIs) of antifungal drugs could provide advantages for dry-powder inhaler-based therapy over liquid nebulizer systems. ${ }^{4}$ DPI-based formulations are solid-state, which provides stability during long-term storage. Moreover, DPI administration time is very short, and the devices to use are portable, less expensive, and require less maintenance than liquid nebulizers. Delivery to the lung is driven by the inspiratory flow and does not require a compressor.

Antifungal drugs, especially azole compounds, possess poor water solubility, which could reduce their efficiency. ${ }^{5}$ After being inhaled and deposited on the pulmonary mucosa, dry active-ingredient particles need to dissolve within the lung surfactant layer to be pharmacologically effective and avoid nonabsorptive clearance mechanisms. ${ }^{6}$ Therefore, solubility of such compounds must be enhanced using a formulation procedure.

One strategy to enhance drug solubility is to reduce the size of the drug particles to the nanometer scale, which greatly increases the specific surface area (area per unit of mass). Therefore, according to the Noyes-Whitney equation, this reduction will increase the drug particle-dissolution velocity. Moreover, reducing particle size also induces surface curvature at the liquid/solid interface. This curvature results in an increase in dissolution pressure and drug solubility, as described with respect to the vapor pressure between a liquid and a gas in the Kelvin and Ostwald-Freudlich equations. ${ }^{7,8}$ If inhaled, these nanoparticles (NP) would not only increase the proportion of drug that is dissolved in the pulmonary lining fluid and is able to act therapeutically but also decrease the proportion of undissolved drug particles that are removed by natural clearance mechanisms.

Pulmonary delivery is dependent on specific variables, such as the particle size. Particles need to possess an aerodynamic diameter $\left(\mathrm{d}_{\mathrm{ae}}\right)$ in the range between 1 and $5 \mu \mathrm{m}$ to reach the lungs. However, administering NP to the lung through a dry-powder inhaler may cause some problems. Particles with a $\mathrm{d}_{\mathrm{ae}}$ below $1 \mu \mathrm{m}$ (in particular, between 0.4 and $0.1 \mu \mathrm{m}$ ) tend to be significantly eliminated during exhalation. ${ }^{9-11}$ The small size of NP causes them to be deposited by diffusion or eliminated during exhalation, instead of being deposited by impaction or sedimentation. Moreover, because of their very large surface area, NP possess strong interparticular forces that reduce dry-material flowability and powder dispersion after emission from a passive dry-powder inhaler. ${ }^{12}$ Because of these physical phenomena, the inhalation of dry NP could result in a very poor fraction of the inhaled dose being deposited in the lung. Therefore, most researchers have studied the delivery of solid NP in microentities, such as droplet dispersion using nebulizers or NP-based agglomerates using dry-powder inhalers. ${ }^{13-16}$ However, NP agglomeration reduces the specific surface area and curvature necessary to increase particle solubility and dissolution rate. Therefore, an alternative formulation strategy is suitable.

The objective of this study was to develop ITZ nanosuspensions (NS) using high-pressure homogenization (HPH) followed by spray-drying in the presence of mannitol, an acceptable filler for inhalation, ${ }^{17}$ to produce mannitol-based microparticles in which ITZ NP are dispersed. This formulation could provide a DPI with a high FPF and enhanced drug solubility while avoiding the general problems encountered during handling and pulmonary delivery of NP.

\section{Materials and methods Materials}

Raw ITZ was purchased from Hetero Drugs (Hyderabad, India). Sodium lauryl sulfate, tocopherol polyethylene 1000 succinate (TPGS), and sodium taurocholate (NaTau) were purchased from Sigma-Aldrich (Steinheim, Germany). Poloxamer 407 was purchased from BASF (Brussels, Belgium). Mannitol (Pearlitol PF) was donated by Roquette Frères (Lestrem, France). All of the solvents used were analytical grade.

\section{Methods}

\section{Formulation preparation}

Two separate steps were performed to produce the formulations. First, an ITZ NS was prepared using HPH. Second, a carrier solution was prepared in which a determined quantity of NP were resuspended. This suspension was further spray-dried to produce the dried particles. The carrier was dissolved in a greater quantity compared to the resuspended NP to encourage the formation of microparticles embedded with NP.

The compositions of the suspensions are summarized in Table 1 . These were prepared by dissolving a quantity of surfactant in a hydroalcoholic solution composed of isopropanol (1/3) and water (2/3) under magnetic stirring (600 rpm) before adding micronized raw ITZ. The "initial suspension" was then homogenized using a CAT high-speed homogenizer (HSH) X620 (CAT Ingenieurbüro, Staufen, Germany) at $24,000 \mathrm{rpm}$ for 10 minutes. This suspension is referred to as 
Table I Initial suspension composition (before spray-drying), theoretical composition of the spray-dried formulation, and ITZ experimental content as determined by HPLC

\begin{tabular}{|c|c|c|c|c|c|c|c|}
\hline Suspension & \multicolumn{2}{|l|}{$\begin{array}{l}\text { ITZ } \\
(\% \text { w/v) }\end{array}$} & \multicolumn{2}{|l|}{$\begin{array}{l}\text { TPGS } \\
(\% \mathrm{w} / \mathrm{v}) \\
\end{array}$} & \multicolumn{3}{|l|}{$\begin{array}{l}\text { TPGS } \\
\left(\% \mathrm{w} / \mathrm{w}_{\mathrm{ITZ}}\right)\end{array}$} \\
\hline \multicolumn{8}{|c|}{ Initial suspension compositions in water/isopropanol (2:I) } \\
\hline SI & 0.33 & & 0.017 & & 5 & & \\
\hline S2 & 0.33 & & 0.033 & & 10 & & \\
\hline S3 & 0.5 & & 0.025 & & 5 & & \\
\hline S4 & 0.5 & & 0.050 & & 10 & & \\
\hline S5 & I & & 0.050 & & 5 & & \\
\hline S6 & 1 & & 0.1 & & 10 & & \\
\hline \multicolumn{5}{|c|}{ DPI theoretical composition after spray-drying } & \multicolumn{3}{|c|}{ Experimental ITZ content } \\
\hline Formulation & $\begin{array}{l}\text { ITZ } \\
(\% w / w)\end{array}$ & $\begin{array}{l}\text { Mannitol } \\
(\% \mathrm{w} / w)\end{array}$ & $\begin{array}{l}\text { TPGS } \\
(\% w / w)\end{array}$ & $\begin{array}{l}\text { NaTau } \\
(\% \text { w/w) }\end{array}$ & $\begin{array}{l}\text { Mean ITZ content } \\
(\% \text { wt) }\end{array}$ & CV (\%) & $\begin{array}{l}\text { Relative error } \\
\text { (\%) }\end{array}$ \\
\hline$\overline{C^{a}}$ & 32.258 & 64.516 & 3.226 & - & 30.6 & 2.8 & -5.2 \\
\hline$N I^{b}$ & 90.91 & - & 9.09 & - & 90.2 & 2.4 & -0.76 \\
\hline $\mathrm{N} 2^{\mathrm{b}}$ & 32.258 & 64.516 & 3.226 & - & 34.1 & 3.8 & 5.71 \\
\hline$N 3^{b, c}$ & 32.154 & 64.309 & 3.215 & 0.322 & 27.4 & 9.4 & -14.9 \\
\hline$N 4^{b, c}$ & 31.949 & 63.898 & 3.195 & 0.958 & 24.1 & 2.02 & -24.5 \\
\hline
\end{tabular}

Notes: aProduced from the initial suspension S6; ${ }^{b}$ produced from the $\mathrm{S} 6$ nanosuspension; ' $\mathrm{NaTau}$ added to the carrier solution before spray-drying.

Abbreviations: CV, coefficient of variation; DPI, dry powder for inhalation; HPLC, high-performance liquid chromatography; ITZ, itraconazole; NaTau, sodium taurocholate; TPGS, tocopherol polyethylene 1000 succinate.

the "prehomogenized suspension." This prehomogenized suspension was then circulated in an EmulsiFlex high-pressure homogenizer (Avestin, Ottawa, Canada) at 20,000 PSI until the minimum particle size to form the homogenized suspension was reached ( 300 cycles). To avoid an increase in sample temperature following the HPH cycles, all operations were carried out using a heat exchanger, which was placed ahead of the homogenizing valve to maintain the sample temperature at $10^{\circ} \mathrm{C} \pm 1{ }^{\circ} \mathrm{C}$. Suspension particle sizes were evaluated during the process using laser light diffraction. After 300 $\mathrm{HPH}$ cycles, $25 \mathrm{~mL}$ of NS was resuspended in $50 \mathrm{~mL}$ of a solution (isopropanol:water 70:30) containing 1\% (w/v) of mannitol. The NSs were spray-dried using a Büchi mini spray-dryer (B-191a; Büchi Laboratory Equipment, Flawil, Switzerland) under the following conditions: spraying air flow $800 \mathrm{~L} /$ hour; drying air flow $35 \mathrm{~m}^{3} /$ hour; solution feed rate $2.7 \mathrm{~g}$ /minute; nozzle diameter $0.5 \mathrm{~mm}$; inlet temperature $80^{\circ} \mathrm{C}$; and outlet temperature $45^{\circ} \mathrm{C}$. This solution/suspension was kept in an ice bath before and after resuspension as well as during the spray-drying process to avoid aggregation and solubilization of the NP.

A control formulation (formulation C) was produced under the exact conditions as those used for the formulations except that a prehomogenized suspension (microparticles in suspension) was added to the carrier solution instead of an NS. One formulation (formulation N1) was spray-dried without the addition of mannitol to the carrier solution (pure spray-dried ITZ NS).

\section{Particle size-distribution analyses}

Particle-size distribution (PSD) analyses were determined by laser light scattering using a Malvern Mastersizer 2000 (Malvern Instruments, Malvern, UK). A wet sample-dispersion unit (Hydro 2000; Malvern Instruments) was used to measure the particle size in the different suspensions during the NS production step and to assess the redispersibility of the NP from the dried formulations. The dispersion medium was composed of ITZ-saturated deionized water containing 1\% Poloxamer 407 to avoid any ITZ particle solubilization and agglomeration. The initial prehomogenized and homogenized suspensions were diluted in the dispersion media for size measurements.

The redispersion abilities of the NP in aqueous media were evaluated by adding and vortex-shaking $10 \mathrm{mg}$ of each formulation in $5 \mathrm{~mL}$ of dispersion media, and the measurement was taken using the Hydro 2000, as described above.

A dry dispersion-sample unit (Sirocco; Malvern Instruments) was used to evaluate the PSD of the dried-state formulations. Tests were performed on samples of approximately $50 \mathrm{mg}$ at a pressure of 4 bars with a feed rate vibration set at $40 \%$. These conditions allowed for the measurement of the PSD of an almost totally deagglomerated powder because of the very drastic dispersion conditions that were generated.

A particle refractive index with a real part of 1.48 and imaginary part of 0.1 was chosen for the formulations, and values of 1.61 and 0.01 were used for the raw ITZ. The reported diameters were calculated using volume distribution and are expressed in terms of $\mathrm{d}(0.1), \mathrm{d}(0.5)$, or median 
particle size, and $\mathrm{d}(0.9)$, which are the sizes in microns at which $10 \%, 50 \%$, and $90 \%$, respectively, of the particles are smaller than the remaining distribution, and as the mean volume particle size $(\mathrm{D}[4,3])$. The results shown are the means \pm standard deviation, determined on the basis of five and three measurements when using the wet- and the dry-dispersion sampling systems, respectively.

\section{Drug-content determinations}

The determinations of the drug contents of the formulations were performed on both the bulk powder (total drug content) and powder that was fractionated following its $\mathrm{d}_{\mathrm{ae}}$ to evaluate if the drug content was similar in each $\mathrm{d}_{\mathrm{ae}}$ fraction.

For the total drug-content determination, an exact quantity of the bulk dry-powder formulation was introduced into a volumetric flask containing the dilution phase and sonicated for 20 minutes. The drug contents were determined from quantification of the solution concentration by a high-performance liquid chromatography ultraviolet (HPLC-UV) method. The procedure was repeated five times for each formulation, and the results are expressed as percentages of the weight of the dry formulation (means \pm standard deviation).

Particle-size separation prior to drug-content determination was accomplished using a next-generation impactor (NGI) (Copley Scientific, Nottingham, England). A hydroxypropyl methylcellulose (HPMC) $n^{\circ} 3$ capsule was filled with a specific quantity of formulation. The powder particles were deposited on the different stages of the NGI after passive emission from an Axahaler device (SMB Laboratories, Brussels, Belgium). This technique allowed for the separation of particles based on their $d_{a e}$. Device emission was accomplished by triggering an airflow of $60 \mathrm{~L} /$ minute for 4 seconds throughout the whole system, which allowed for the collection of particles with $\mathrm{d}_{\mathrm{ae}}$ between 8.06 and $4.46 \mu \mathrm{m}, 4.46$ and $2.82 \mu \mathrm{m}, 2.82$ and $1.66 \mu \mathrm{m}, 1.66$ and $0.94 \mu \mathrm{m}, 0.94$ and $0.55 \mu \mathrm{m}$ on stages $2,3,4,5$, and 6 , of the impactor respectively. Determination of the drug content was then conducted on these different aerodynamic fractions of particles using the same procedure as that used for the bulk content determination. The drug contents of the different powder fractions were expressed as the percentages of the exact mean drug concentration calculated for the bulk powder (total drug content), which allows for comparisons between formulations.

\section{Crystallinity evaluation}

The crystallinity properties of the different materials and formulations were assessed using both differential scanning calorimetry (DSC) and powder X-ray diffraction (PXRD). DSC analyses were conducted using a Q 2000 DSC (TA Instruments, Zellik, Belgium). Samples (2-3 mg) were placed in sealed aluminum hermetic pans. The temperature was increased from $25^{\circ} \mathrm{C}$ to $180^{\circ} \mathrm{C}$ with a heating rate of $10^{\circ} \mathrm{C} /$ minute under a nitrogen gas flow $(50 \mathrm{~L} /$ minute $)$. PXRD analyses were performed using a Siemens D5000 diffractometer (Siemens, Ville, Germany) with a $K \alpha$ line of copper with monochromatic radiation $(\lambda=1.540 \AA)$ at a voltage of $40 \mathrm{kV}$ and a current of $40 \mathrm{~mA}$. Samples were scanned with an angular $2 \theta$ range from $2^{\circ}$ to $60^{\circ}$ in steps of $0.02^{\circ}$, with a counting speed of 1.2 seconds per step and a sample rotation speed of $15 \mathrm{rpm}$.

\section{Morphology evaluation}

The morphology of the particles was observed with an environmental scanning electron microscope (XL30 ESEM-FEG; Philips, Eindhoven, Netherlands) after gold coating. The acceleration voltage during the observations ranged from $5 \mathrm{keV}$ to $25 \mathrm{keV}$, depending on the samples. Images were taken randomly at magnifications ranging from $1000 \times$ to $20,000 \times$. Prior to imaging, the samples were spread on a carbon adhesive band, then coated with gold at $40 \mathrm{~mA}$ for 90 seconds at $6 \times 10^{2}$ mbar under argon to a thickness of approximately $15-20 \mathrm{~nm}$.

\section{Powder flowability}

Powder flowability was evaluated by determining the Carr index (CI) for each formulation. A preweighed quantity of dry powder was placed in a graduated $10-\mathrm{mL}$ cylinder. The apparent volume occupied by the powder was then noted before and after application of 1000 taps to the cylinder using a tap density tester (Stampfvolumeter STAV 2003; Engelsmann, Ludwigshafen, Germany). The CI is a function of the difference between the tapped and bulk densities expressed as a percentage of the tapped density.

\section{Aerodynamic properties}

The theoretical primary $d_{a e}$ of the particles (which is an estimation of the $d_{a e}$ of the single particles) was calculated based on the following equation:

$$
d_{a e}=d_{0.5} \sqrt{\frac{t_{p}}{\rho}} \text { where } \rho=1 \mathrm{~g} / \mathrm{cm}
$$

where $d_{0.5}$ is the median particle size as determined by laser diffraction $(\mathrm{d}(0.5))$ and $t_{p}$ is the powder tapped density. ${ }^{18}$ 
The in vitro lung deposition of each formulation after actuation from the Axahaler dry-powder inhaler was assessed using a multistage liquid impinger (Copley Scientific, Nottingham, England). A flow rate (adjusted to a pressure drop of $4 \mathrm{kPa}$ ) of $100 \mathrm{~L} /$ minute was applied for 2.4 seconds throughout the device for each actuation. The device was filled with $\mathrm{HPMC} \mathrm{n}{ }^{\circ} 3$ capsules loaded with a quantity of dry powder corresponding to $2.5 \mathrm{mg}$ of ITZ. Each test was performed with three capsules. After the three dose actuations, the total ITZ deposited in each part of the impactor was quantified by a suitable and validated HPLC-UV method. ${ }^{5}$ Each test was replicated three times. For each test, the FPF and the mass median aerodynamic diameter (MMAD) were estimated by the method described in the European Pharmacopeia 7.2 for the aerodynamic assessment of fine particles using apparatus $\mathrm{C}$ (multistage liquid impinger). The FPF is the dose of particles presenting a $\mathrm{d}_{\mathrm{ae}}$ below $5 \mu \mathrm{m}$, expressed as a percentage of the emitted dose. The MMAD is the $\mathrm{d}_{\mathrm{ae}}$ of the particles deposited in the impactor at which $50 \%(\mathrm{w} / \mathrm{w})$ of particles have a lower and $50 \%(\mathrm{w} / \mathrm{w})$ have a higher $\mathrm{d}_{\mathrm{ae}}$.

\section{Supersaturation evaluation in a physiological $\mathrm{pH}$ phosphate buffer}

A physiological $\mathrm{pH}$ phosphate buffer with ( $\mathrm{pH}$ of 7.2, European Pharmacopoeia 7.2) was used to evaluate the ITZ solubility and was composed (w/v) of $0.8 \%$ sodium chloride, $0.02 \%$ potassium chloride, $0.01 \%$ calcium chloride, $0.01 \%$ magnesium chloride, $0.318 \%$ disodium hydrogen phosphate, and $0.02 \%$ potassium dihydrogen phosphate. Dipalmitoylphosphatidylcholine $(0.02 \% \mathrm{w} / \mathrm{v})$ was added to this solution, as described by Son and $\mathrm{McCo}-$ nville, to simulate the wetting effect of lung surfactant. ${ }^{19,20}$ Before testing, an NGI was used - as described in the "Aerodynamic properties" section - to collect particles that presented $\mathrm{a}_{\mathrm{ae}}$ between 2.82 and $4.46 \mu \mathrm{m}$, which were then introduced into the physiological media. The tests were performed using a USP 33 type II (paddle method) dissolution apparatus (Distek, North Brunswick, $\mathrm{NJ}$ ) equipped with $100 \mathrm{~mL}$ glass-dissolution vessels and small rotating paddles (the rotating speed was set to 200 $\mathrm{rpm})$. In the dissolution tests, excess powder formulation was prewetted in $2 \mathrm{~mL}$ of dissolution medium $\left(4^{\circ} \mathrm{C}\right)$ by vortex shaking for 5 seconds and introduced into $100 \mathrm{~mL}$ of dissolution medium that was maintained at $37.0^{\circ} \mathrm{C} \pm 0.2^{\circ} \mathrm{C}$. Subsequently, $2 \mathrm{~mL}$ samples were withdrawn after $2,5,10,15,30,45,60,75,90,120$ and 180 and 180 minutes and were directly filtered through $0.1 \mu \mathrm{m}$ filters. The samples were reconstituted with acetonitrile for HPLC-UV quantification, and the tests were performed in triplicate.

\section{HPLC-UV analytical method}

The ITZ determination was performed using a HPLC-UV method, as described elsewhere. ${ }^{5}$

\section{Results and discussion \\ Nanosuspension production}

The initial goal of formulation manufacturing was to produce a NS using HPH. This procedure for top-down size reduction was chosen both because of its recognized applications in the size reduction and production of NP of poorly water-soluble drugs and for its ability to be easily scaled up for industrial production. ${ }^{21-23}$

The use of a surfactant is necessary in the preparation of NP for the stabilization of the crystals in suspension after they are broken in the homogenizing valve. ${ }^{22}$ The surfactant minimizes particle agglomeration, which improves the size-reduction process. The surfactant type and concentration as well as the concentration and hardness of the active ingredient are the primary parameters that influence the sizereduction process. ${ }^{22}$ The arbitrarily chosen values for NP size in this study were $0.25 \mu \mathrm{m}$ and $2 \mu \mathrm{m}$ for $\mathrm{d}(0.5)$ and $\mathrm{d}(0.9)$, respectively, which correspond to the common minimal particle size range reached using a top-down size-reduction process approach such as $\mathrm{HPH} .{ }^{24}$ A preliminary study was performed to select the appropriate surfactant to stabilize the suspension in different media. TPGS was selected as the surfactant and water-isopropanol $(2 / 1 \mathrm{v} / \mathrm{v})$ as the dispersant medium. Potential surface-active agents were chosen because of their low-potential pulmonary toxicity (bile salts, phospholipids, and cholesterol). ${ }^{25}$ However, none of these agents, even when associated in different proportions, allowed the particle size to be reduced below $2 \mu \mathrm{m}$ (data not shown). Reduction of the proportion of isopropanol considerably affected particle wettability, inducing poor powder dispersion and severe agglomeration in the liquid. This poor dispersion systematically caused blocking of the homogenizing valve, which interfered with the process. From the selected homogenization dispersion medium, the concentrations of ITZ were investigated at $0.33 \%, 0.5 \%$, and $1 \%(\mathrm{w} / \mathrm{v})$ with $5 \%$ or $10 \%$ TPGS (expressed as a percentage of ITZ weight) (Table 1). The targeted size values $(\mathrm{d}(0.5)<0.25 \mu \mathrm{m}, \mathrm{d}(0.9)<2 \mu \mathrm{m})$ were only obtained with suspension S6 (ie, the higher ITZ and TPGS concentrations), which was then chosen for production of the dry formulations. Higher ITZ concentrations with the TPGS ratio maintained at 10\% (expressed as a percentage 
of ITZ weight) were tested, but with problems of blocking the homogenizing valve. Higher TPGS ratios were not tested because of its low melting point $\left(\sim 42^{\circ} \mathrm{C}\right)$, which could soften and melt during the spray-drying process and imply particle agglomeration.

The PSD after each stage of the size-reduction process for suspension S6 are detailed in Table 2. The initial suspension showed very high particle sizes $(\mathrm{D}[4,3]$ of $5.91 \mu \mathrm{m}$ and $\mathrm{d}(0.9)$ of $11.19 \mu \mathrm{m})$ that were superior to those of raw ITZ as measured by laser diffraction in the dry mode (raw ITZ; D[4,3] of $3.17 \mu \mathrm{m}$ and $\mathrm{d}(0.9)$ of $5.72 \mu \mathrm{m}$, Table 3$)$. As observed by SEM (Figure 1), raw ITZ is composed of shale-like plate crystal sheets surrounded by smaller irregular crystals. The high-energy dispersion conditions used during particle-size measurements in the dry mode can remove those finer crystals, which are therefore measured as individualized particles, and partly erode the fragile crystal sheets, which are measured to be smaller than they are. During the initial size measurement of the suspension in the wet mode, those smaller particles were in the form of agglomerates and gave superior size values. For the prehomogenized particle sizes, the energetic dispersion using the HSH $(24,000 \mathrm{rpm}$ for 10 minutes) was sufficient to disperse the fine particles, which were thereafter stabilized by the TPGS in solution. Moreover, the PSD values for the prehomogenized suspension are slightly lower than for the original dry material, which indicates a moderate size reduction after homogenization by the $\mathrm{HSH}$. In contrast, the application of $300 \mathrm{HPH}$ cycles led to the production of an NS as we previously defined it containing particles presenting a PSD with a $d(0.5)$ and a $d(0.9)$ of $0.221 \mu \mathrm{m}$ and $1.676 \mu \mathrm{m}$, respectively. The homogenized suspension presented two very distinct populations of particles of approximately $200 \mathrm{~nm}$ and $1.1 \mu \mathrm{m}$, although more than $50 \%$ of particles were below $220 \mathrm{~nm}$ in this suspension. This second population was assumed to result from NP agglomeration and/or remaining microparticles. ${ }^{15,22}$

\section{Formulation characterization}

Formulations N1, N2, N3, and N4 were produced from the homogenized NS S6. Unlike formulations N2, N3, and N4, formulation N1 was spray-dried without mannitol in the solution (ie, it was a purely spray-dried NP formulation), which allowed the effect of mannitol on the dry-particle properties to be evaluated. Moreover, formulation $\mathrm{C}$ was produced from the initial suspension S6 (ie, micronized ITZ in suspension) with the addition of mannitol and TPGS in the same proportions as those for formulation $\mathrm{N} 2$ to evaluate the impact of the size-reduction process on the properties of the dry particles. Formulations N3 and N4 were produced to evaluate the effect of adding an endogenous charged surfactant to the formulation.

\section{Physicochemical properties}

PXRD results (Figure 2) showed that the homogenization operations and the spray-drying process did not affect the ITZ crystalline state. Indeed, formulation N1 was obtained after the spray-drying of an NS without mannitol, and the initial ITZ diffraction profile was clearly recovered on its diffractogram. However, the ITZ diffraction rays from formulation N1 were of lower intensity than those observed from the raw crystalline ITZ. This difference may be caused by particle-size reduction and/or the dilution of the NP in the surfactant (TPGS), which most likely coated the particle. ${ }^{22}$ The lack of amorphous domains and the conservation of the ITZ crystalline state after the entire process were confirmed by DSC. Raw crystalline ITZ melted at a temperature of $167.8^{\circ} \mathrm{C}$, while formulation $\mathrm{N} 1$ melted at a slightly lower temperature $\left(164.9^{\circ} \mathrm{C}\right)$. Because

Table 2 Laser diffraction diameters (Malvern Mastersizer 2000 Hydro) of suspension S6 during the different stages of the sizereduction process and of the spray-dried formulations C, NI, N2, N3, and N4 after reconstitution in aqueous media

\begin{tabular}{lcccc}
\hline & $\begin{array}{l}\mathrm{d}(\mathbf{0 . 1}) \\
(\mu \mathrm{m})\end{array}$ & $\begin{array}{l}\mathrm{d}(\mathbf{0 . 5}) \\
(\mu \mathrm{m})\end{array}$ & $\begin{array}{l}\mathrm{d}(\mathbf{0 . 9}) \\
(\mu \mathrm{m})\end{array}$ & $\begin{array}{l}\mathrm{D}[\mathbf{4 , 3}] \\
(\mu \mathrm{m})\end{array}$ \\
\hline Malvern Mastersizer Hydro 2000 & & & & \\
Initial suspension & $2.07 \pm 0.01$ & $4.79 \pm 0.01$ & $11.19 \pm 0.05$ & $5.91 \pm 0.03$ \\
Prehomogenized suspension & $1.47 \pm 0.04$ & $2.69 \pm 0.02$ & $4.95 \pm 0.08$ & $2.99 \pm 0.02$ \\
Homogenized suspension (100 cycles) & $0.100 \pm 0.007$ & $1.08 \pm 0.08$ & $2.6 \pm 1.0$ & $1.6 \pm 0.9$ \\
Homogenized suspension (300 cycles) & $0.083 \pm 0.001$ & $0.221 \pm 0.010$ & $1.676 \pm 0.007$ & $0.630 \pm 0.009$ \\
C & $2.220 \pm 0.002$ & $5.15 \pm 0.01$ & $12.6 \pm 0.1$ & $6.7 \pm 0.2$ \\
N1 & $1.33 \pm 0.02$ & $2.43 \pm 0.01$ & $4.65 \pm 0.07$ & $2.780 \pm 0.003$ \\
N2 & $0.098 \pm 0.001$ & $1.51 \pm 0.01$ & $2.47 \pm 0.01$ & $1.15 \pm 0.01$ \\
N3 & $0.091 \pm 0.001$ & $0.78 \pm 0.02$ & $2.0 \pm 1.0$ & $0.90 \pm 0.01$ \\
N4 & $0.084 \pm 0.0004$ & $0.25 \pm 0.01$ & $1.77 \pm 0.01$ & $0.653 \pm 0.009$ \\
\hline
\end{tabular}




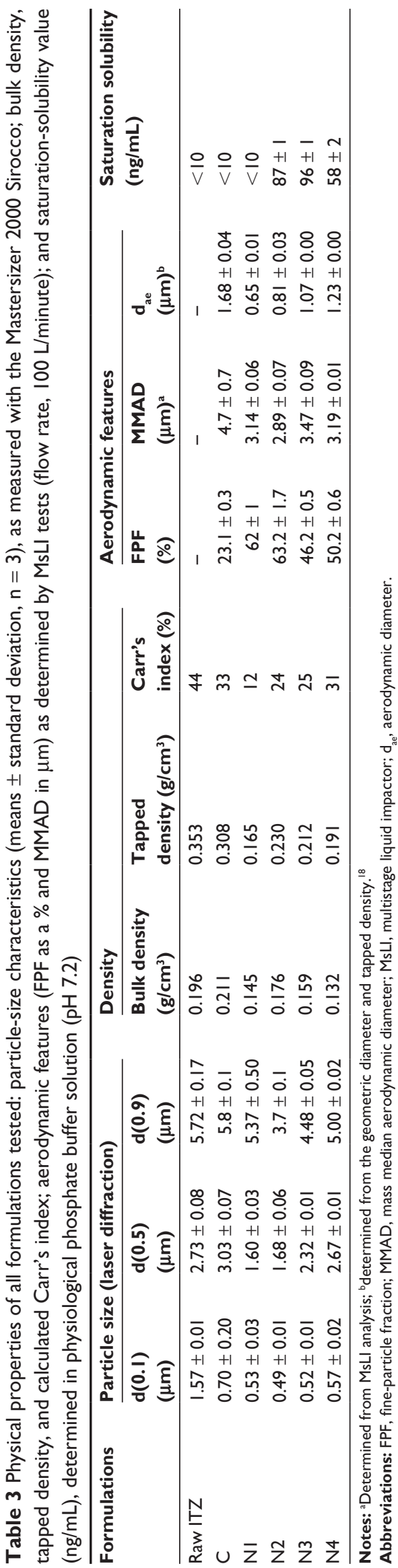

the crystallinity was confirmed by PXRD and a thermal event characteristic of amorphous ITZ was not observed, ${ }^{5}$ this lower melting temperature was attributed to a smaller crystalline size, which may cause a lower melting point. Spray-dried mannitol was found to be crystalline, as shown by its diffractogram. In all formulations, an endothermic event was present at approximately $37^{\circ} \mathrm{C}$, corresponding to the melting point of quench-cooled TPGS, which originally melted at $42^{\circ} \mathrm{C}$ and was therefore assumed to be in the amorphous phase in the spraydried formulations. On the diffractograms of formulations C, N2, N3, and N4, the specific identification of the different constituents of the formulations was more complicated than for formulation N1 because of the presence of many diffraction peaks that overlapped with those of mannitol, ITZ, TPGS, and NaTau. However, some diffraction rays that are characteristic of crystalline ITZ (at $8.69^{\circ} 2 \theta, 12.29^{\circ} 2 \theta$, and $16.12^{\circ} 2 \theta$ ) can be specifically detected on the diffractograms of the different spray-dried formulations, as shown in Figure 2. These rays confirm the presence of crystalline ITZ in the four formulations. The conservation of ITZ and mannitol crystallinity may present advantages for the long-term stability of the product.

Analysis of the particle size after reconstitution of the dried formulations in aqueous media (Table 2) revealed that the presence or absence of mannitol during the spray-drying of homogenized suspension S6 considerably affected the NP aggregation state. Severe aggregation of the NP occurred if the surfactant was spray-dried in the absence of this excipient, as indicated by the tremendous increase in particle size for formulation N1. This formulation showed a d $(0.5)$ of $2.43 \mu \mathrm{m}$, whereas the NS before spray-drying had a d $(0.5)$ of $0.22 \mu \mathrm{m}$. Even after 5, 15, or 30 minutes of sonication, these aggregates were not disrupted, and the measured particle size remained unchanged, which indicates an irreversible aggregation. In the scanning electron microscope micrograph of formulation N1 (Figure 1), the presence of large agglomerates and aggregates is clear. These aggregates may be formed from NP during the drying step as a result of the capillary forces acting on the particles during liquid evaporation. ${ }^{26} \mathrm{NP}$ aggregation was reported by Van Eerdenburgh et al to be more pronounced for highly hydrophobic surface compounds, such as ITZ. ${ }^{27}$ Additionally, TPGS, which is present in a relatively significant proportion ( $10 \% \mathrm{w} / \mathrm{w}$ of ITZ), could have created semisolid bridges between particles (TPGS melting point is $\sim 42^{\circ} \mathrm{C}$ ) that would induce agglomeration. The addition of NaTau impeded the agglomeration of NP in a quantity-dependent manner. Formulations N3 and N4 contained $1 \%$ and 3\% of NaTau (\% w/w of ITZ), respectively. The PSD parameters (Table 2) after 


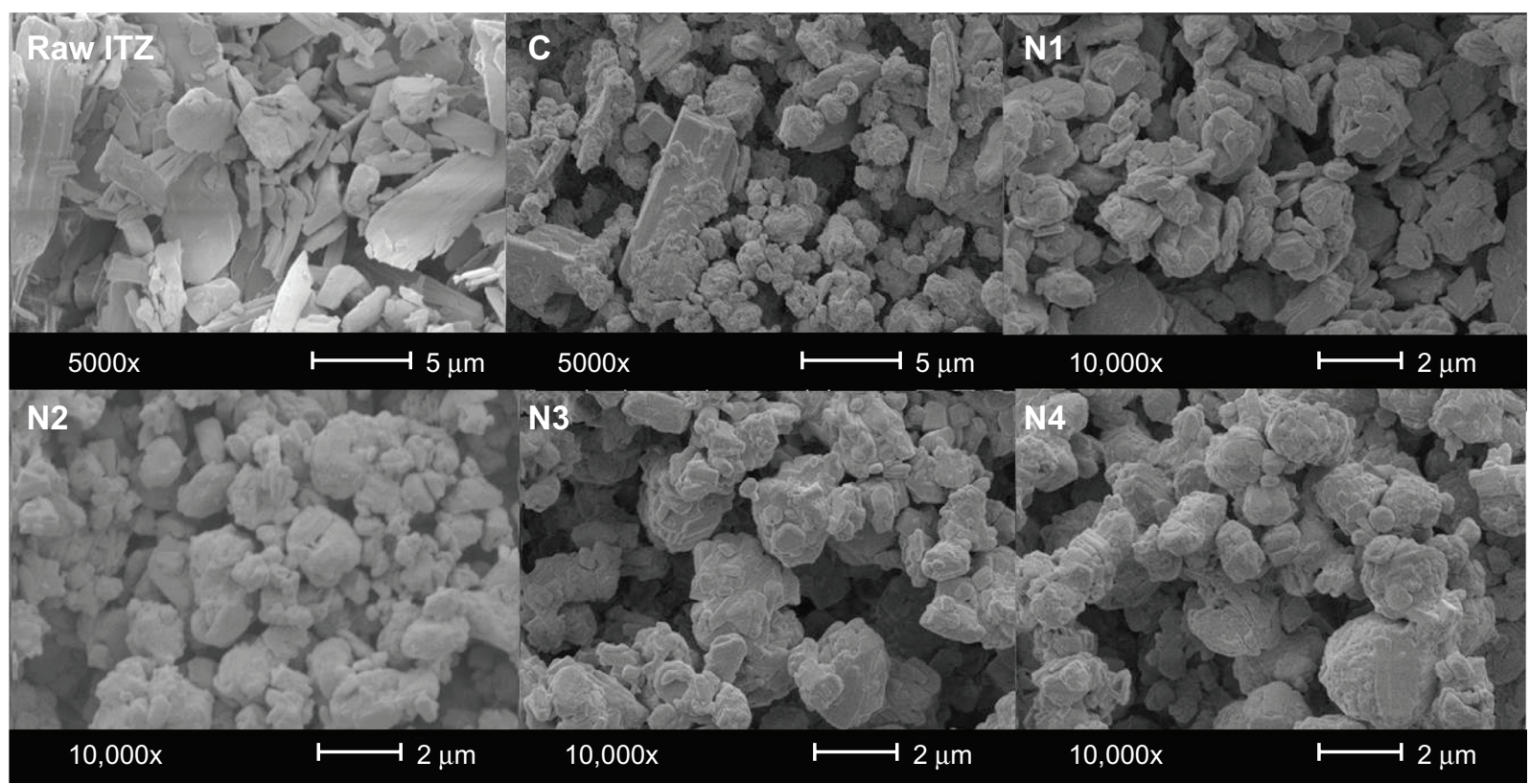

Figure I Scanning electron microscope micrographs of raw itraconazole (ITZ) and the spray-dried formulations at magnifications of 5000× and I0,000×.

reconstitution in the aqueous media of formulation N4 were close to those of the original NS, whereas the particle sizes for formulation $\mathrm{N} 3$ were greater (ie, $\mathrm{d}(0.5)$ of $0.22,0.78$, and $0.25 \mu \mathrm{m}$ for the original NS and formulations N3 and N4, respectively). This inhibition of particle agglomeration might be caused by surface coating of the NP by the negatively charged surfactant NaTau. Such a coating would have created particle repulsions during spray-drying and inhibited the irreversible aggregation of particles. ${ }^{28}$ However, $1 \%$ of NaTau (w/w of ITZ) seemed to insufficiently cover the entire surface of the particles and did not provide a sufficiently large charge load on the particle surfaces. Therefore, a light agglomeration still

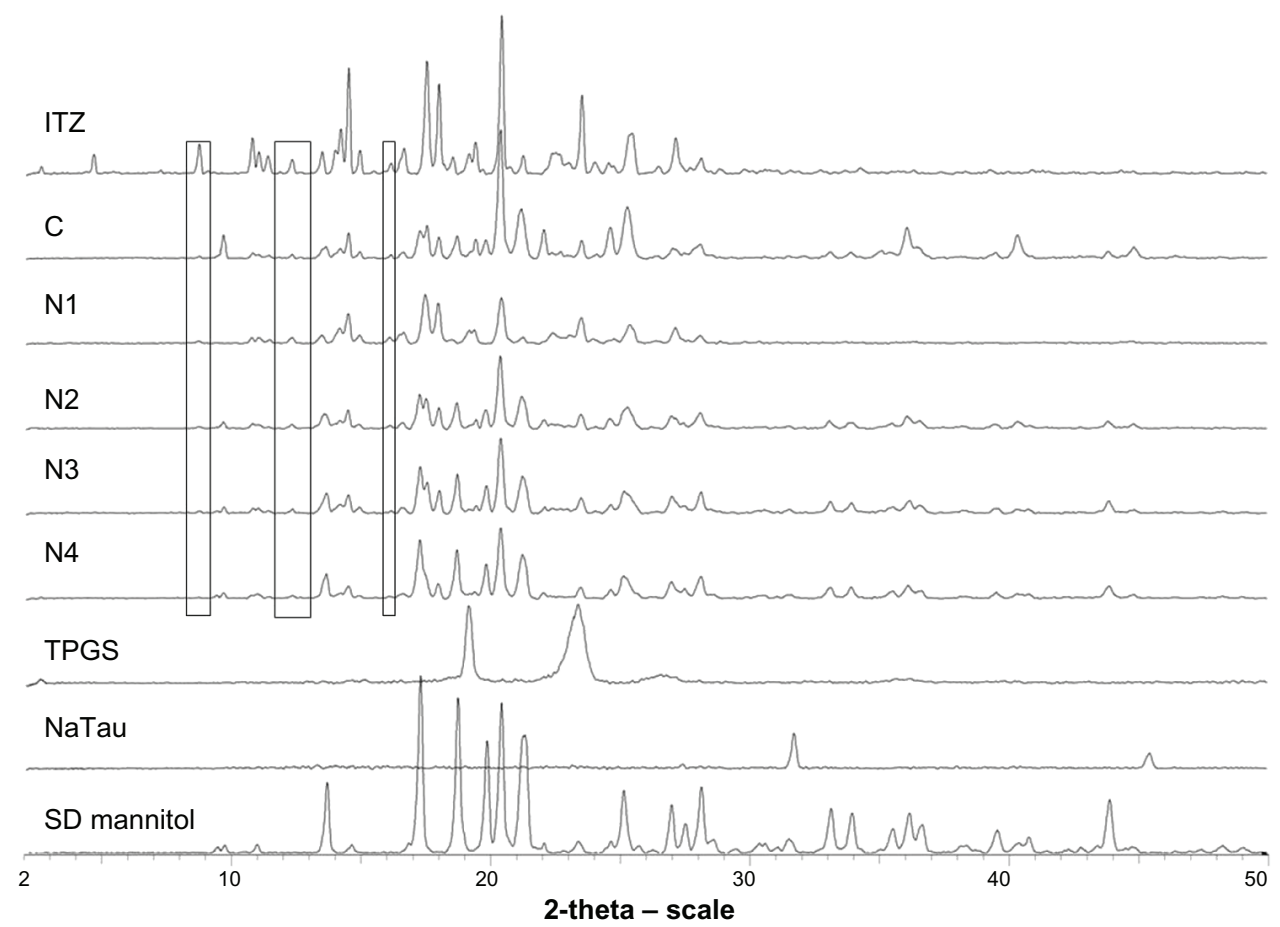

Figure 2 Powder X-ray diffractograms of raw itraconazole (ITZ), formulations C, NI, N2, N3, and N4, and excipients tocopherol polyethylene I000 succinate (TPGS), sodium taurocholate ( $\mathrm{NaTau})$, and spray-dried (SD) mannitol. 
occurred in formulation N3. When the NaTau concentration was increased to $3 \%(\mathrm{w} / \mathrm{w}$ of ITZ), the concentration was adequate, and the size distribution of the spray-dried particles remained unchanged (Table 2). Therefore, given that all of the particle sizes measured by laser diffraction in the dry mode were in the micrometer range $(\mathrm{d}(0.5)$ values from $1.68 \mu \mathrm{m}$ to $2.67 \mu \mathrm{m}$, Table 3 ), the NP or aggregated NP were likely to be embedded correctly in the mannitol microparticles.

The measured total drug contents were consistent with the expected theoretical values for formulations $\mathrm{C}, \mathrm{N} 1$, and N2, which exhibited very low relative errors (Table 1). However, formulations N3 and N4 presented relative errors greater than $10 \%$ (Table 1 ), which can be explained by the loss of active ingredient during the spray-drying process consecutively to NP surface accumulation. Indeed, after nebulization of the NS from the nozzle of the spray-dryer, NP are assumed to be distributed uniformly in the produced droplets. ${ }^{8,29}$ On one hand, when the droplets were formed from the nozzle of the spray-dryer, surface tension at the air/liquid interface of each droplet was created, and NP are known to accumulate at this interface to reduce surface tension. ${ }^{30}$ On the other hand, during the fast drying of droplets, NP migrate to the extreme edge of the droplets, which is called the "coffee-stain effect." 31 Indeed, the drying of a droplet is not instantaneous but a rapid progressive phenomena during which the droplet shrinks at a determined radial velocity from its outer edge to its center. The internal distribution of material that is dissolved or suspended in a droplet in the final dried product can be determined by the ratio between droplet evaporation rate and their diffusional motion, ${ }^{32}$ which can be expressed by the Peclet number $(P e)$. The $P e$ is a dimensionless mass-transport number that compares the times for droplet drying and suspended/dissolved particle diffusion and is defined by the following equation: $P e=\mathrm{R}^{2} /(\mathrm{td} \mathrm{D})$, where $\mathrm{R}$ is the radius of the droplet, $\mathrm{td}$ is the time required for a droplet to dry, and $\mathrm{D}$ is the solute (or NP) diffusion coefficient. ${ }^{33}$ For $P e<<1$ (slow droplet evaporation, small droplet, or high diffusion mobility), solute or NP within the droplet have sufficient time to redistribute throughout the evaporating droplet; therefore, material is homogeneously distributed with the dried particle. By contrast, for $P e>>1$ (rapid evaporation, low diffusion coefficient, large droplet), solutes or NP have insufficient time to diffuse from the surface to the center of the droplet, and as a result the particle surfaces are enriched by the component with the high $P e$. When our particles were spray-dried, two major components where present in the droplets, the mannitol and the suspended ITZ NP. Mannitol is considered to be a low Pe compound, ${ }^{34}$ whereas NP in suspensions typically have extremely high $P e$ numbers, because compared to the receding droplet surface the suspended NP can be considered as immobile. ${ }^{32}$ These two previously described features consequently induce NP accumulation at the surface of the dried material. As previous results have indicated, the degree of aggregation of NP during drying gradually decreases with the addition of NaTau. Therefore, for the formulations in

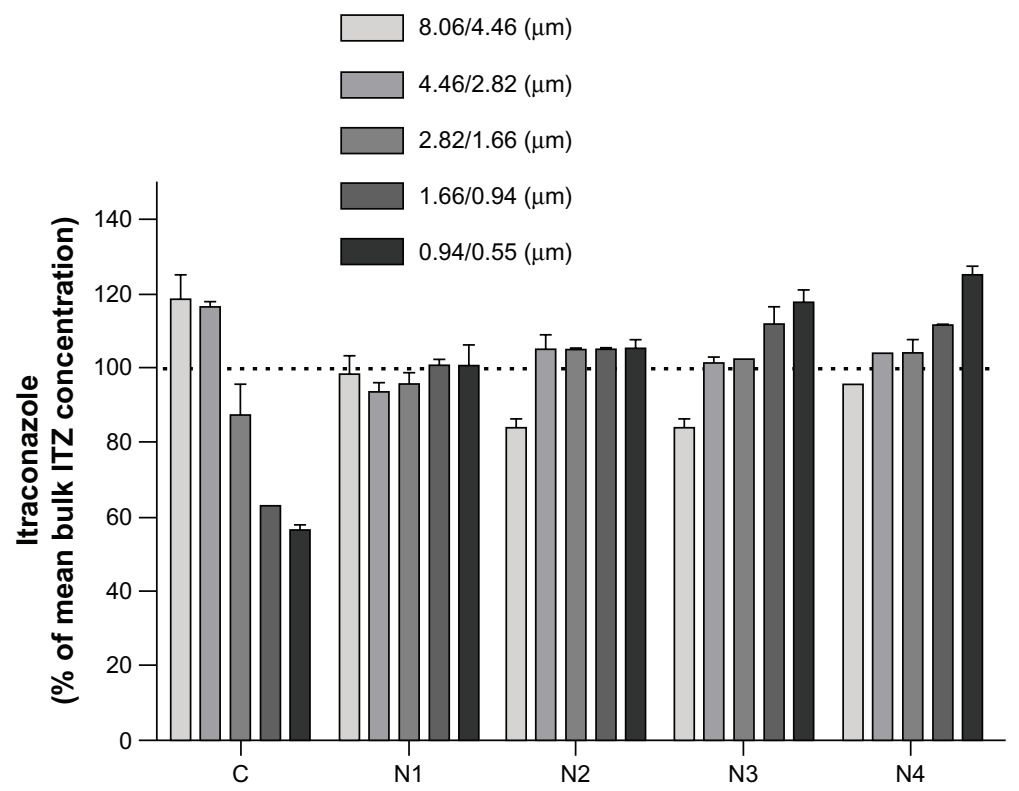

Figure 3 Graphical representation of formulations' itraconazole (ITZ) content (expressed as a \% of the mean experimental total ITZ content) for each aerodynamic particlesize fraction.

Note: Powders were fractionated in a next-generation impactor at $60 \mathrm{~L} /$ minute. 
which NP agglomeration was impeded (formulation N3 and even more so with formulation N4), the NP at the surfaces of the dried particles were smaller than those of the formulation without NaTau (formulation N1). These smaller particles possess a greater probability of being eroded or detached during the spray-drying procedure because of their greater specific surface areas and the resulting higher probability of interaction compared to larger particles. Once detached from the core particles, these smaller particles could either be driven by the airflow of the spray-dryer and lost in the filter collector or remain in the cyclone with the rest of the powder. The fraction that was removed by the airflow was the reason for the loss of active ingredient. Simultaneously, the fraction that remained in the cyclone and collector most likely resulted in the increase in drug content for the very fine particles of formulations N3 and N4, as observed in the dosing drug content as a function of $\mathrm{d}_{\mathrm{ae}}$. Indeed, Figure 3 represents the drug content, expressed as the percentage of drug content measured for bulk powder, determined for the different powder $\mathrm{d}_{\mathrm{ae}}$ fractions of each formulation. For formulations $\mathrm{N} 3$ and N4, the drug content increased as the particles became smaller. This correlation was clearer for formulation N4, which possessed the smallest ITZ particles on the surface of the microparticles. Formulation N2, which was composed of mannitol and NP with a higher degree of aggregation, did not show this specific drug-distribution profile. Formulation N1, which was composed of irreversible aggregates of NP, possessed an ITZ content that was similar for each $\mathrm{d}_{\mathrm{ae}}$ fraction of powder (approximately 100\% of the bulk content). This result was expected because of the simple composition of this formulation (no matricial agent). As shown in Figure 3 for formulation C, the drug contents of the aerodynamic size fractions decreased in concert with reductions in the particle aerodynamic size. This result was also expected because smaller nebulized droplets from the nozzle of the spray-dryer are not able to encapsulate larger ITZ crystals after the solidification of mannitol. This behavior is easily observed in Figure 1, where the typical larger plate sheet shale-like crystals of raw ITZ are not completely incorporated into mannitol crystalline microparticles.

The CI values allow for the powder flowability to be evaluated. Values of less than $25 \%$ usually indicate good flowability, whereas values above $40 \%$ are associated with very poor flowability. Raw ITZ showed very poor flowability (CI of $44.5 \%$, Table 3 ), which can be explained by the very irregular shape of these particles (Figure 1), which favors particle interaction and interlocking that is caused by the numerous interaction sites on the surface. The N1, N2, N3, and N4 formulation particles possessed a more homogenous and regular shape than the raw ITZ and formulation $\mathrm{C}$, which most likely improved the powder flowability (CI of $12 \%, 24 \%, 25 \%$, and $31 \%$ for formulations N1, N2, N3, and N4, respectively, versus CI of $44.5 \%$ and $33 \%$ for raw ITZ and formulation C, respectively). Formulation N1 (ie, pure spray-dried NS) showed the best flowability, which can be explained by the conversion of the $\mathrm{NP}$ in the suspension into microparticles (D[4,3] of $2.51 \mu \mathrm{m})$ during the spray-drying process. The reduction of the ITZ crystal size was, despite the combination of the NP with mannitol microparticles, associated with an increase in CI that resulted in decreased powder flowability. The ITZ crystal size of formulations N2, N3, and N4 progressively decreased from $1.15 \mu \mathrm{m}$ to $0.653 \mu \mathrm{m}$, which gradually increased the CI from $23.5 \%$ to $31 \%$. Indeed, the NP tended to accumulate at the mannitol surfaces and enhance the particle-specific surfaces following the reduction in NP size. In contrast, the presence of NaTau particle surfaces could create interparticular repulsions, which are unfavorable for powder flowability. However, the powder-flow characteristics of these formulations were still acceptable because the flow is dominated by the size of the microparticles and not by the size of the NP that are embedded in the microparticles. Practically, the dry-powder formulations flowed easily and did not stick at all in the glass vial. One particular aspect that could also be considered is the presence of TPGS in all formulations, which has previously been shown to improve powders flowability by a supposed lubricant effect. ${ }^{5}$

\section{Evaluation of particle size and in vitro lung deposition}

The aim of inhalation therapy for invasive pulmonary aspergillosis is to maximize the concentration of the antifungal drug in the lung and minimize the systemic level of exposure, which is higher with intravenous or oral administration. After dose inhalation from an inhaler device, the dry powders require the proper aerodynamic characteristics to reach the sites of fungal invasion. ${ }^{35}$ The lung tissue of the host can only be invaded in regions of the lung where the spores have been deposited and germinated. The deposition sites are determined by the $\mathrm{d}_{\mathrm{ae}}$ of the Aspergillus spores that present a $\mathrm{d}_{\mathrm{ae}}$ reported to be between 1.9 and $6 \mu \mathrm{m}$, depending on the particular study, Aspergillus species, and spore-sampling methods. ${ }^{36-38}$ The theoretical $\mathrm{d}_{\mathrm{ae}}$ values determined from the median particle size and particle tapped density for each formulation are reported in Table 3 and were below the targeted d $\mathrm{a}_{\mathrm{ae}}$ of Aspergillus conidia (1.9-6 $\left.\mu \mathrm{m}\right)$. However, after dispersion from an inhaler, aerosol particles are generally not inhaled as single particles because the surface 


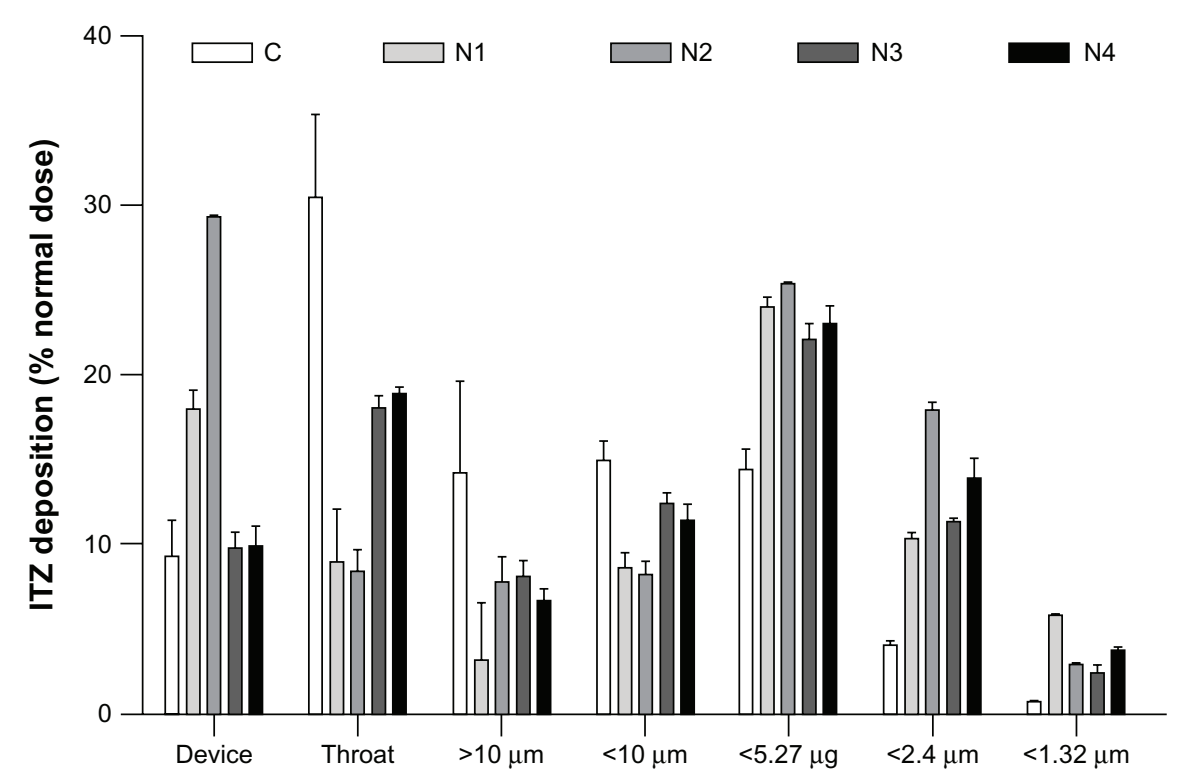

Figure 4 Graphical representation of the in vitro pulmonary deposition patterns of the C, NI, N2, N3, and N4 formulations (MsLI, I00 L/minutes, 2.4 seconds, $\mathrm{n}=3$ ). Note: Itraconazole (ITZ) doses were recovered from the device, the throat, and stages I-5 of the multistage liquid impactor deposition and are expressed as a \% of the nominal dose $(2.5 \mathrm{mg})$.

interactions of the particles impede total dispersion. Instead, they are inhaled as agglomerates, which generally increase their $\mathrm{d}_{\mathrm{ae}} \cdot{ }^{39}$ Consequently, particle-dispersion properties, combined with their size and density, influence the aerosol $\mathrm{d}_{\mathrm{ae}}$ during inhalation and the resulting deposition sites. For these reasons, the aerodynamic properties of our dry-powder aerosol formulations generated from a dry-powder inhaler in simulated breathing conditions were evaluated and characterized by their MMAD, which represented the $\mathrm{d}_{\mathrm{ae}}$ of the aerosol, and by the in vitro FPF, which represents the percentage of the emitted dose that possesses a $\mathrm{d}_{\mathrm{ae}}<5 \mu \mathrm{m}$, and are reported in Figure 4 and Table 3 . The in vitro FPF is a good indicator of pulmonary drug targeting because the lower respiratory tract is almost always the primary focus of infection that results from inhalation of the infectious spores. ${ }^{40}$

Formulation $\mathrm{C}$ showed the poorest aerodynamic performance, with a very low FPF (23.1\%) and the highest MMAD $(4.7 \pm 0.7 \mu \mathrm{m}$, Table 3$)$. This formulation was produced by spray-drying a prehomogenized microsuspension of ITZ with dissolved mannitol. The ITZ in suspension possessed a mean particle size of approximately $3 \mu \mathrm{m}$. Therefore, when the particles were formed after solvent evaporation, the mannitol solidified and entrapped large ITZ particles, which enhanced the final particle diameter through the addition of mannitol "subunits" to the larger crystals, as illustrated in Figure 1. In addition, because of the large size of the ITZ particles, the smallest mannitol particles contained lower concentrations of ITZ (Figure 3), which reduced the amount of active ingredi- ent that reached the lower stages of the impactor (Figure 4). Very poor disaggregation and dispersion properties for this formulation were observed under simulated breathing conditions using a dry-powder inhaler because more than $50 \%$ of the emitted dose of formulation $\mathrm{C}$ was stopped in the induction port and the first stage of the impactor, which stopped particles having a $\mathrm{d}_{\mathrm{ae}}>10 \mu \mathrm{m}$, even though the $\mathrm{d}_{\mathrm{ae}}$ of the primary theoretical particle was estimated to be $1.68 \pm 0.04 \mu \mathrm{m}$. Formulation N2 possessed the same qualitative composition as formulation C (Table 1), but exhibited a 2.7 -fold higher FPF (FPF of $23.1 \%$ for formulation C vs $63 \%$ for formulation N2) and a lower MMAD (2.89 $\mu \mathrm{m}$ and $4.7 \mu \mathrm{m}$ for formulations $\mathrm{N} 2$ and $\mathrm{C}$, respectively). The improved aerodynamic behavior of formulation $\mathrm{N} 2$ was most likely a result of its smaller particle size compared with that of formulation $\mathrm{C}(\mathrm{d}(0.5)$ of $1.68 \mu \mathrm{m}$ and $3.03 \mu \mathrm{m}$ for formulations $\mathrm{N} 2$ and $\mathrm{C}$, respectively), which is caused by the smaller ITZ crystals that were better distributed between the mannitol microparticles. Furthermore, formulation N2 showed a more regular spherical shape in the SEM than formulation C, which exhibited irregular morphology with extensive flat surfaces that may promote a large contact area and would result in increased adhesion between the particles. ${ }^{41}$ This increase in adhesion may have reduced the redispersion abilities of the powder and the agglomeration of particles, which should also have increased the MMAD of the aerosol. Finally, the tapped density of formulation $\mathrm{C}$ was higher than that of formulation $\mathrm{N} 2\left(0.308 \mathrm{~g} / \mathrm{cm}^{3}\right.$ and $0.230 \mathrm{~g} / \mathrm{cm}^{3}$ for formulations $\mathrm{C}$ and 
$\mathrm{N} 2$, respectively), which also increased the primary $\mathrm{d}_{\mathrm{ae}}$ of the particles and the MMAD of formulation $\mathrm{C}$.

Formulation $\mathrm{N} 1$ and $\mathrm{N} 2$ aerodynamic behaviors were similar, with FPF of $62 \%$ and $63 \%$, respectively. However, the particles of the $\mathrm{N} 1$ and $\mathrm{N} 2$ formulations possessed a theoretical primary $\mathrm{d}_{\mathrm{ae}}$ of $0.65 \mu \mathrm{m}$ and $0.85 \mu \mathrm{m}$, whereas their experimental MMAD values reversed and showed values of $3.14 \pm 0.06 \mu \mathrm{m}$ and $2.89 \pm 0.07 \mu \mathrm{m}$, respectively. This result indicates that the particles of formulation N1 had a lower deagglomeration efficiency compared to those of formulation N2, despite the lower density. This lower density counterbalanced the higher density of formulation N2, and aerosolized particles with a close MMAD value were produced. When comparing the composition of the particles, formulation N1 is composed solely of ITZ NP aggregates and TPGS, while formulation N2 is composed of ITZ NP aggregates and TPGS diluted in mannitol. During the production of formulation N1, the TPGS was dissolved in the spray-dried ITZ NS, which may have resulted in the formation of a coating of the TPGS agent around the ITZ-aggregated NP. ${ }^{22}$ Because of its waxy and sticky composition, the TPGS was most likely responsible for the poor deagglomeration efficiency. This effect was most likely attenuated in formulation $\mathrm{N} 2$ because of the lower concentration of TPGS $(9.09 \%$ of weight of dry product in formulation N1 vs $3.226 \%$ in formulation N2), which was a result of dilution of TPGS within the mannitol in the final dry form.

The addition of NaTau progressively increased the particle sizes. The formulations N2 ( $0 \%$ NaTau, w/w ITZ), N3 ( $1 \% \mathrm{NaTau})$, and $\mathrm{N} 4$ (3\% NaTau) showed $\mathrm{d}(0.5)$ values of
$1.68 \mu \mathrm{m}, 2.32 \mu \mathrm{m}$, and $2.67 \mu \mathrm{m}$, respectively. As expected from these PSD analysis results, the FPF of formulations N3 and N4 were reduced compared with those of formulation N2 (FPF of $46.2 \%$ and $52.2 \%$ for formulations N3 and N4, respectively, vs $63.2 \%$ for formulation N2). The higher particle size enhanced the theoretical primary $d_{a e}$ of the particles of formulations N3 and N4 compared with that of formulation N2 (Table 3). As is clearly shown in Figure 4, a considerably higher proportion of formulations N3 and N4 was stopped in the induction port compared with formulation N2 (ie, corresponding to particles with $\mathrm{a}_{\mathrm{ae}}$ more than ten times greater than the primary $\mathrm{d}_{\mathrm{ae}}$ of the particle), which suggests lower particle deagglomeration. These two formulations contained NaTau, which is a negatively charged surfactant. During powder dispersion, electrostatic charges are commonly generated from the dry-powder inhaler. ${ }^{42}$ Therefore, NaTau charges may promote interaction between those charges, which reduces particle dispersion. Despite a higher particle size and higher $\mathrm{d}_{\mathrm{ae}}$ than formulation $\mathrm{N} 3$, formulation N4 had a higher FPF (46.2\% and 50.2\% for formulations N3 and N4, respectively) because of the higher quantity of active ingredient that reached the last three stages of the impactor. This difference may be a result of the smallest particles of these two formulations being composed of a high percentage of ITZ NP (Figure 3). Therefore, these particles constituted a smallest size fraction for formulation $\mathrm{N} 4$, which could reduce the $\mathrm{d}_{\mathrm{ae}}$ of this size fraction because of their higher degree of porosity.

The MMAD values of the formulation aerosols (from $2.89 \mu \mathrm{m}$ for formulation N2 to 3.47 for formulation N3, Table 3 ) in simulated breathing conditions from an inhaler device corresponded well to the aerodynamic values of

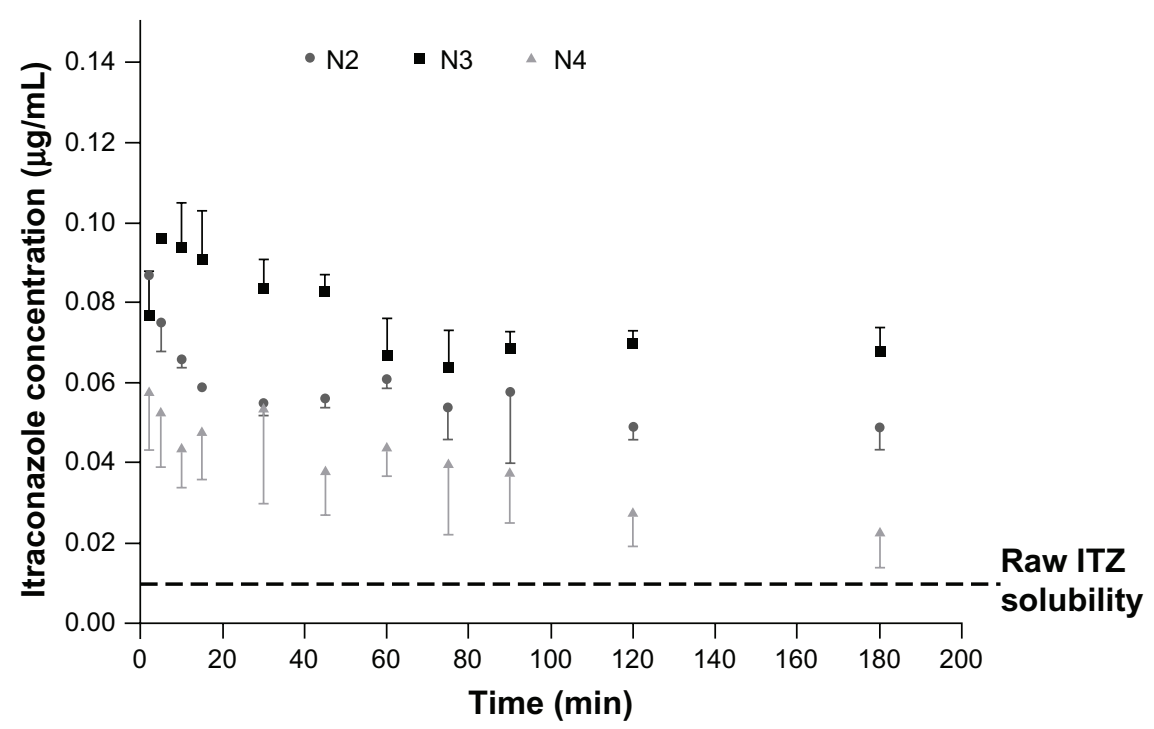

Figure 5 Supersaturation solubility of formulations N2, N3, and N4 in physiological phosphate buffer (pH 7.2) containing 0.02\% dipalmitoylphosphatidylcholine. Abbreviation: ITZ, itraconazole. 
potentially inhaled Aspergillus conidia (between $1.9 \mu \mathrm{m}$ and $6 \mu \mathrm{m}$ ). These antifungal drug-based dry-powder formulations should allow the Aspergillus infection sites in lung mucosa to be reached effectively.

\section{Dissolution in supersaturation conditions}

The solubility of ITZ in simulated lung fluid has been reported to be extremely low $(\sim 8-10 \mathrm{ng} / \mathrm{mL}) \cdot{ }^{43}$ Therefore, increasing the solubility of ITZ could improve supersaturation of the pulmonary lining fluid. Supersaturation of the pulmonary lining fluid around the fungal infiltration region may increase the fraction of active ingredient that is absorbed into fungal cells and able to inhibit the fungal cytochrome $\mathrm{p} 450$, which is involved in the essential ergosterol biosynthesis of aspergilli. ${ }^{4}$ Moreover, it has previously been demonstrated that the increase in ITZ particle solubility has a direct positive impact on antifungal in vivo activity after administration direct into the lung. ${ }^{43,45}$ To determine the supersaturation levels of the different formulations, dissolution tests were performed on a powder fraction corresponding to their MMAD. Their ITZ saturation-solubility profile as a function of time is illustrated in Figure 5.

During the solubility tests, media ITZ concentrations for the raw ITZ and formulations C and N1 were below the detection limit (ie, $\sim 10 \mathrm{ng} / \mathrm{mL}$ ). In contrast, formulations $\mathrm{N} 2$, N3, and N4 exhibited high saturation solubility with maximum experimentally determined values of $87 \pm 1 \mathrm{ng} / \mathrm{mL}$, $96 \pm 1 \mathrm{ng} / \mathrm{mL}$, and $58 \pm 2 \mathrm{ng} / \mathrm{mL}$, respectively (Table 3), which are at least six to ten times higher than the raw ITZ and formulations $\mathrm{C}$ and $\mathrm{N} 1$. These results revealed that the NS must be spray-dried in the presence of mannitol to allow for significant improvement in the solubility (formulations $\mathrm{N} 2$, N3, and N4 versus formulation N1). Unexpectedly, the best improvements in saturation solubility were provided by particles having a median diameter of 0.78 and $1.51 \mu \mathrm{m}$ (formulations N2 and N3). To explain this enhancement in saturation solubility, the increase in particle-surface curvature induced by the particle-size reduction cannot be taken, as it only exponentially increases below a particle size of $100 \mathrm{~nm}$. Regarding the Ostwald-Freundlich equation, enhancing particle-surface curvature should have induced an increase in particle-surface tension $(\gamma)$ that consecutively increased particle solubility. Therefore, given ITZ particle sizes were higher than $100 \mathrm{~nm}$, other factors must be taken into account to explain solubility enhancement. It has been reported that an increase in $\gamma$ (therefore in solubility) can also take place during sample processing, such as during a particle size-reduction procedure by high-pressure homogenization. The very energetic conditions encountered during this type of procedure might lead to an increase in $\gamma$ and thus to an increase in saturation solubility. ${ }^{46}$ Converting microparticles into NP might also lead to the formation of defects in the original crystals. These crystal defects, including dislocations, influence the crystal lattice energy and give rise to increased surface energy and thus to an increased saturation solubility. ${ }^{47,48}$ It was supposed that the formulations spray-dried in the presence of mannitol provided higher saturation levels of ITZ compared to bulk or pure spray-dried NP because of these two probable facts. The agglomerated ITZ NP in formulations N2 and N3 were probably composed of small ITZ subunits that aggregated after the spray-drying but that still presented their ability to supersaturate the phosphate buffer during the saturation test. The hydrophilic nature of the mannitol also allowed a good dispersion of ITZ particles in the dissolution media, which can be problematic for a very hydrophobic compound such as ITZ. These kinds of structures also allowed enhancement of ITZ-specific surface area compared to the ITZ original microstructured. Noteworthy, formulation N4 possessed the smallest ITZ crystals (Table 2) and the lowest solubility (Table 3) compared with formulations N2 and N3. This low solubility is caused by the instability of the NP in aqueous media. ${ }^{49}$ This instability was observed by laser diffraction during particle-size measurements after reconstitution in the dissolution media that was saturated in ITZ, at a very high stirring rate over time (at ambient temperature). When adding the prewetted formulations (ITZ NP in suspension), a new population of approximately $10-\mu \mathrm{m}$ particles was observed, and the original NP population decreased over time. These changes indicated NP agglomeration or Ostwald ripening in the dissolution media, which were likely to be more pronounced as the particle size decreased. For the saturationsolubility profile over time (Figure 5), maximum saturation solubility was reached quickly and thereafter decreased to a lower solubility value caused by agglomeration and crystal maturation. Formulation N3, with an intermediate size of ITZ crystal particles, seemed to present the most suitable size characteristics to maximize solubility.

\section{Conclusion}

Spray-drying ITZ NS in the presence of mannitol was an effective method of producing dry powders with good aerosolization properties and high FPF values, and promoted the formation of supersaturated solutions of ITZ. The proposed method may constitute an interesting alternative for the treatment or for the prophylaxis of invasive pulmonary aspergillosis because the effective antifungal dose, which we 
previously estimated to be $1.2 \mathrm{mg}$ (corresponding to $3-4 \mathrm{mg}$ of dry-powder formulations) to overcome resistance, ${ }^{35}$ could be directly administered deep into the lung, where it could saturate the lining fluid, which has previously been shown to enhance ITZ in vivo activity after inhalation. ${ }^{43,45}$

\section{Disclosure}

The authors report no conflicts of interest in this work.

\section{References}

1. Hope WW. Invasion of the alveolar-capillary barrier by Aspergillus spp.: therapeutic and diagnostic implications for immunocompromised patients with invasive pulmonary aspergillosis. Med Mycol. 2009; 47 Suppl 1:S291-S298.

2. Pfaller MA, Diekema DJ. Epidemiology of invasive mycoses in North America. Crit Rev Microbiol. 2010;36:1-53.

3. Le J, Schiller DS. Aerosolized delivery of antifungal agents. Curr Fungal Infect Rep. 2010;4:96-102.

4. Labiris NR, Dolovich MB. Pulmonary drug delivery. Part II: the role of inhalant delivery devices and drug formulations in therapeutic effectiveness of aerosolized medications. Br J Clin Pharmacol. 2003;56: 600-612.

5. Duret C, Wauthoz N, Sebti T, Vanderbist F, Amighi K. Solid dispersions of itraconazole for inhalation with enhanced dissolution, solubility and dispersion properties. Int J Pharm. 2012;428:103-113.

6. Mobley C, Hochhaus G. Methods used to assess pulmonary deposition and absorption of drugs. Drug Discov Today. 2001;6:367-375.

7. Müller RH, Jacobs C, Kayser O. Nanosuspensions as particulate drug formulations in therapy. Rationale for development and what we can expect for the future. Adv Drug Deliv Rev. 2001;47:3-19.

8. Rabinow BE. Nanosuspensions in drug delivery. Nat Rev Drug Discov. 2004;3:785-796.

9. Sung JC, Pulliam BL, Edwards DA. Nanoparticles for drug delivery to the lungs. Trends Biotechnol. 2007;25:563-570.

10. Sham JO, Zhang Y, Finlay WH, Roa WH, Lobenberg R. Formulation and characterization of spray-dried powders containing nanoparticles for aerosol delivery to the lung. Int J Pharm. 2004;269:457-467.

11. Yang W, Peters JI, Williams RO 3rd. Inhaled nanoparticles - a current review. Int J Pharm. 2008;356:239-247.

12. Weers JG. Dispersible powders for inhalation applications. Innov Pharm Technol. 2000;1:111-116.

13. El Gendy N, Berkland C. Combination chemotherapeutic dry powder aerosols via controlled nanoparticle agglomeration. Pharm Res. 2009; 26:1752-1763.

14. El Gendy N, Gorman EM, Munson EJ, Berkland C. Budesonide nanoparticle agglomerates as dry powder aerosols with rapid dissolution. J Pharm Sci. 2009;98:2731-2746.

15. Pilcer G, Vanderbist F, Amighi K. Preparation and characterization of spray-dried tobramycin powders containing nanoparticles for pulmonary delivery. Int $J$ Pharm. 2009;365:162-169.

16. Salem H, Abdelrahim M, Eid KA, Sharaf M. Nanosized rods agglomerates as a new approach for formulation of a dry powder inhaler. Int $J$ Nanomedicine. 2011;6:311-320.

17. Daviskas E, Anderson SD, Jaques A, Charlton B. Inhaled mannitol improves the hydration and surface properties of sputum in patients with cystic fibrosis. Chest. 2010;137:861-868.

18. Hinds WC. Aerosol Technology: Properties, Behavior, and Measurement of Airborne Particles. 2nd ed. New York: Wiley; 1999: 42-47.

19. Son YJ, McConville JT. Development of a standardized dissolution test method for inhaled pharmaceutical formulations. Int J Pharm. 2009; 382:15-22.

20. Pham S, Wiedmann TS. Note: dissolution of aerosol particles of budesonide in Survanta, a model lung surfactant. J Pharm Sci. 2001;90: 98-104.
21. Van Eerdenbrugh B, Van den MG, Augustijns P. Top-down production of drug nanocrystals: nanosuspension stabilization, miniaturization and transformation into solid products. Int J Pharm. 2008;364:64-75.

22. Hecq J, Deleers M, Fanara D, Vranckx H, Amighi K. Preparation and characterization of nanocrystals for solubility and dissolution rate enhancement of nifedipine. Int J Pharm. 2005;299:167-177.

23. Keck CM, Muller RH. Drug nanocrystals of poorly soluble drugs produced by high pressure homogenisation. Eur J Pharm Biopharm. 2006;62:3-16.

24. Van Eerdenbrugh B, Van den Mooter G, Augustijns P. Top-down production of drug nanocrystals: nanosuspension stabilization, miniaturization and transformation into solid products. Int J Pharm. 2008;364: 64-75.

25. Pilcer G, Amighi K. Formulation strategy and use of excipients in pulmonary drug delivery. Int J Pharm. 2010;392:1-19.

26. Wang B, Zhang W, Zheng W, Mujumdar AS, Huang L. Progress in drying technology for nanomaterials. Drying Technol. 2004;23: 1-18.

27. Van Eerdenbrugh B, Froyen L, Van Humbeeck J, Martens JA, Augustijns P, Van den Mooter G. Drying of crystalline drug nanosuspensions - the importance of surface hydrophobicity on dissolution behavior upon redispersion. Eur J Pharm Sci. 2008;35:127-135.

28. Chaubal MV, Popescu C. Conversion of nanosuspensions into dry powders by spray drying: a case study. Pharm Res. 2008;25: 2302-2308.

29. Chan HK, Gonda I. Development of a systematic theory of suspension inhalation aerosols. 2. Aggregates of monodisperse particles nebulized in polydisperse droplets. Int J Pharm. 1988;41:147-157.

30. Binks BP. Particles as surfactant - similarities and differences. Curr Opin Colloid Interface Sci. 2002;7:21-41.

31. Majumder M, Rendall C, Eukel A, Wang J, Behabtu Natnael, Pint C, Liu T, Orbaek A, Mirri F, Nam J.. Barron A, Hauge R, Schmidt H, Pasquali M, Overcoming the "Coffee-Stain" Effect by Compositional Marangoni-FlowAssisted Drop-Drying, J Phys chem; 2012; 116:6536-6542.

32. Tsapis N, Bennett D, Jackson B, Weitz D, Edwards DA. Trojan particles: large porous carriers of nanoparticles for drug delivery. Proc Natl Acad Sci U S A. 2002;99:12001-12005.

33. Stroock AD, Dertinger SK, Ajdari A, Mezic I, Stone HA, Whitesides GM. Chaotic mixer for microchannels. Science. 2002;295: 647-651.

34. Hao J, Li SK. Mechanistic study of electroosmotic transport across hydrated nail plates: effects of $\mathrm{pH}$ and ionic strength. J Pharm Sci. 2008; 97:5186-5197.

35. Duret C, Wauthoz N, Sebti T, Vanderbist F, Amighi K. New respirable and fast dissolving itraconazole dry powder composition for the treatment of invasive pulmonary aspergillosis. Pharm Res. 2012, 29(10):2845-59.

36. Morris G, Kokki MH, Anderson K, Richardson MD. Sampling of Aspergillus spores in air. J Hosp Infect. 2000;44:81-92.

37. Eduard W. Fungal spores: a critical review of the toxicological and epidemiological evidence as a basis for occupational exposure limit setting. Crit Rev Toxicol. 2009;39:799-864.

38. Pasqualotto AC. Differences in pathogenicity and clinical syndromes due to Aspergillus fumigatus and Aspergillus flavus. Med Mycol. 2009;47 Suppl 1:S261-S270.

39. Weiler C, Egen M, Trunk M, Langguth P. Force control and powder dispersibility of spray dried particles for inhalation. J Pharm Sci. 2010;99:303-316.

40. Soubani AO, Chandrasekar PH. The clinical spectrum of pulmonary aspergillosis. Chest. 2002;121:1988-1999.

41. Chew NY, Tang P, Chan HK, Raper JA. How much particle surface corrugation is sufficient to improve aerosol performance of powders? Pharm Res. 2005;22:148-152.

42. Byron PR, Peart J, Staniforth JN. Aerosol electrostatics. I: Properties of fine powders before and after aerosolization by dry powder inhalers. Pharm Res. 1997;14:698-705.

43. Yang W, Johnston KP, Williams RO 3rd. Comparison of bioavailability of amorphous versus crystalline itraconazole nanoparticles via pulmonary administration in rats. Eur J Pharm Biopharm. 2010;75: 33-41.

44. Denning DW, Hope WW. Therapy for fungal diseases: opportunities and priorities. Trends Microbiol. 2010;18:195-204.

45. Zhang J, Wu L, Chan HK, Watanabe W. Formation, characterization, and fate of inhaled drug nanoparticles. Adv Drug Deliv Rev. 2011;63: 441-455. 
46. Peters K, Kruss B, Becker R, Müller RH. Pharmaceutical nanosuspensions for medicament administration as systems with increased saturation solubility and rate of solution. United States patent US005858410A. January 12, 1999.

47. Müller RH, Peters K. Nanosuspensions for the formulation of poorly water soluble drugs. I. Preparation by a size reduction technique. Int J Pharm. 1998;160:229-237.
48. Lee RW, Shaw JM, McShane J, Wood RW. Particle size reduction. In: Liu R, editor. Water-Insoluble Drug Formulation. Denver: Interpharm Press; 2000:455-492.

49. Kayaert P, Anne M, Van den MG. Bead layering as a process to stabilize nanosuspensions: influence of drug hydrophobicity on nanocrystal reagglomeration following in-vitro release from sugar beads. J Pharm Pharmacol. 2011;63:1446-1453.
International Journal of Nanomedicine

\section{Publish your work in this journal}

The International Journal of Nanomedicine is an international, peerreviewed journal focusing on the application of nanotechnology in diagnostics, therapeutics, and drug delivery systems throughou the biomedical field. This journal is indexed on PubMed Central, MedLine, CAS, SciSearch $\AA$, Current Contents ${ } /$ Clinical Medicine,

\section{Dovepress}

Journal Citation Reports/Science Edition, EMBase, Scopus and the Elsevier Bibliographic databases. The manuscript management system is completely online and includes a very quick and fair peer-review system, which is all easy to use. Visit http://www.dovepress.com/ testimonials.php to read real quotes from published authors.

Submit your manuscript here: http://www.dovepress.com/international-journal-of-nanomedicine-journal 